\title{
Daily step-count and change in waist circumference during a workplace pedometer program
}

\author{
Kathryn Backholer ${ }^{*}$, Rosanne Freak-Poli ${ }^{\#}$, Anna Peeters \\ Department of Epidemiology \& Preventive Medicine, Faculty of Medicine, Nursing \& Health Sciences, School of Public Health and \\ Preventive Medicine, Monash University, Melbourne, Australia; ${ }^{*}$ Corresponding Author: kathryn.backholer@bakeridi.edu.au
}

Received 9 January 2012; revised 18 February 2012; accepted 20 March 2012

\section{ABSTRACT}

Background: The health benefit associated with a daily step-count target within pedometer programs is unclear. The aim of this study was to determine if the daily step-count attained during a four month pedometer-based workplace health program was associated with change in waist circumference (WC). Methods: 762 Australian adults enrolled in a workplace pedometer program were recruited from ten workplaces in 2008. At the end of the program (four months), 436 participants were eligible for the current analysis. Data included demographics, perceived physical activity change during the program, measured WC at baseline and follow-up, and reported daily pedometer step-counts throughout the program. The association between daily step count and change in WC was examined using linear regression. Results: WC improved by an average of $-1.61 \mathrm{~cm}(95 \% \mathrm{Cl}:-2.13,-1.09)$ by the end of the program. There was no relationship between daily step-count and the degree of change in WC. However, among participants reporting an increase in physical activity during the program a relationship between daily step count and change in WC was observed, such that those who undertook on average 10,000 steps or more per day improved their WC by $-1.38 \mathrm{~cm}(95 \% \mathrm{Cl}:-2.14$, -0.63) more than those who did not achieve an average of 10,000 steps per day. Similarly, among individuals not meeting WC guidelines at baseline a greater daily step count was associated with a greater decrease in WC. Conclusions: Within a workplace pedometer program, reported daily step count was not associated with greater reductions in WC. However, it was a useful indicator of potential health benefits in those who increased their level of physical activity during

\footnotetext{
${ }^{\#}$ These authors contributed equally.
}

the program. Pedometer programs need to communicate clearly the importance of both a step goal and improvement in step count to manage participant expectations about improvements in health markers.

Keywords: Prevention; Health Promotion; Intervention; Physical Activity; Sedentary; Pedometer; Steps; Step Count

\section{INTRODUCTION}

With the rise of obesity and its sequelae there has been an increase in the number of interventions to promote physical activity. The World Health Organisation has recognised the important role of the workplace in promoting health and physical activity [1]. The workplace offers a unique environment for reaching a large number of individuals at once and has been shown to be an effective site for the implementation of health promotion activities that increase the physical activity of employees [2].

The use of pedometers to encourage walking presents a low cost method that enables participants to increase physical activity by keeping track of the number of steps accumulated daily [3]. A growing body of evidence suggests that workplace walking interventions using pedometers can positively influence physical activity behaviour [4] and improve health outcomes such as adiposity measures and blood pressure [5-9]. Accordingly, the primary motivation for participating in such programs appear to be for health reasons [9]. A common goal of pedometer-based interventions is the achievement of 10,000 steps per day $[10,11]$, yet little evidence underpins this benchmark [8]. In order to achieve the maximal health benefits associated with these workplace pedometer-based programs, and thus meet the expectations of participating employees, we need to understand whether achieving 10,000 steps per se is associated with improved health outcomes. 
A before and after analysis of a four month pedometer-based physical activity workplace health program, the Global Corporate Challenge ${ }^{\circledR}$, demonstrated significant improvements in physical activity levels and waist circumference (WC) associated with the program. The current analysis represents a post-hoc analysis of whether the average number of daily steps reported for individuals enrolled in the workplace health program was directly related to the degree of change in $\mathrm{WC}$ at completion of the four month program. In addition, we hypothesised that a higher step count is more likely to be associated with health benefits among those who enter the program at high risk, and among individuals who increase physiccal activity levels during the program. Consequently, we analysed whether any observed relationship between daily step count and change in WC differed according to baseline $\mathrm{WC}$ and perceived change in physical activity during the program.

\section{METHODS}

\subsection{Study Population}

Melbourne workplaces were approached to be study sites for an evaluation of the $\mathrm{GCC}^{\circledR}$ workplace health program. Employees from those workplaces who had already registered for the $\mathrm{GCC}^{\circledR} 2008$ Event were then contacted to participate. Voluntary participants were enrolled in the $\mathrm{GCC}^{\circledR}$ Evaluation Study if they were 18 years of age or over, participating in the $2008 \mathrm{GCC}^{\circledR}$ and at a participating workplace. 762 eligible participants were recruited from ten Melbourne workplaces in early 2008 with $79 \%$ returning to participate at the four month follow-up data collection $(n=604)$. Participants who attended the follow-up survey but had missing values for WC at baseline or follow-up $(n=204)$, who were pregnant at either baseline or follow-up $(n=13)$, had less than 100 out of a possible 125 recorded step entries $(n=$ 90), or who were missing information on perceived change in PA $(n=62)$ were excluded from the analysis, leaving 436 participants. Additional information about the study population can be found elsewhere $[9,12]$.

\subsection{Description of the Program}

The Global Corporate Challenge ${ }^{\circledR}\left(\mathrm{GCC}^{\circledR}\right)$ is a corporate organisation that undertakes a four month, pedometer-based, physical activity health program in the workplace. The GCC $^{\circledR}$ occurs worldwide annually with teams of 7 employees, including a team leader. The aim is to take at least 10,000 steps per day, with teams combining their steps to virtually walk around a world map. Emails are sent to participants on a weekly basis to encourage continued participation and remind them to use the website for logging their daily steps.

\subsection{Data Collection}

Physical measurements were collected by trained health practitioners during morning scheduled appointments. Figure Finder Tape Measures (Novel Products Inc 2005 code PE024) were used to measure WC to the nearest $0.1 \mathrm{~cm}$ with mirrors to ensure the tape was horizontal. To ensure correct midway measurement of the waist was recorded, a picture and instructions were used to ask participants to point out their lower rib margin and the top of the hip (iliac crest). WC measurements were dichotomised by guideline recommendations of less than $94 \mathrm{~cm}$ for males and $80 \mathrm{~cm}$ for females [13]. A self-reported survey was collected over the internet to obtain information on demographics and behavioural measures. Seven options were available for education ranging from "No formal training" to "Post graduate degree", which were dichotomised to those with or without tertiary education. Due to the lack of baseline step data, and therefore the inability to calculate actual change in step count for each individual, change in physical activity levels were estimated by a self-reported questionnaire to determine the change in overall physical activity levels as a result of the health program. Perceived change in physiccal activity was assessed at completion of the workplace health program by asking participants "During the Global Corporate Challenge ${ }^{\circledR}$ (the past 4 months) my activity level was:" with answer options "less than previously", "about the same as previously" or "more than previously". The pedometers were manufactured by the GCC $^{\circledR}$, specifically for the GCC $^{\circledR} 2008$ Event. The pedometers were small, light, portable, easy-to-use electronic devices that counted the number of steps taken by an individual who was informed to have it clipped to the their clothing at the hip. An internal validity process was undertaken where test pedometers were attached to a mechanical instrument imitating a stepping movement.

\subsection{Calculation of Daily Step Count}

Throughout participation in the workplace health program individuals were given pedometers to wear during waking hours to record the daily number of steps taken. As part of the $\mathrm{GCC}^{\circledR}$ program, participants were asked to enter the steps taken at the end of each day into an online step diary. If participants were unable to record their steps, for reasons such as "sick", "travel", "broken" (pedometer), or "forgotten" (pedometer), daily steps were estimated by the workplace health program as the participants current average step count minus $10 \%$.

For each individual, reported daily step count during the $\mathrm{GCC}^{\circledR}$ was calculated to represent the likely actual step count achieved per day. In the primary analysis, reported daily steps was calculated to include all esti- 
mated steps other than those that were due to "sick" reasons and was calculated by dividing the total steps recorded over the four month workplace health program (minus the "sick" estimated steps) by 125 days. The effect of using alternative step data (including either all estimated steps or no estimated steps from missing entries) to calculate daily steps was examined.

Daily step count was analysed as a continuous variable, dichotomised into less than 10,000 steps or equal to or greater than 10,000 steps, and according to the step-defined physical activity hierarchy described by TutorLocke and Bassett [14] as sedentary ( $<5000$ step/day) or below active (collapsed into one category due to low numbers; 5000 - 7499 steps/day), somewhat active (7500 - 9999 steps/day), active (10,000 - 12,499 steps/day) or highly active ( $>12,500$ steps/day).

\subsection{Analysis}

Descriptive statistics were carried out to describe the study population. The main relationship of interest was between reported daily step count during the program and four month change in WC. Assessment was first undertaken with reported daily step count and change in WC as continuous variables. Secondly, the relationship between step categories (dichotomised and step-defined physical activity hierarchy) and change in WC was assessed. Thirdly, the effect of stratifying according to WC guidelines at baseline on the relationship between reported daily step count and WC change was assessed. Finally, the effect of stratifying according to perceived change in physical activity during the workplace health program on the relationship between reported daily step count and WC change was assessed. Linear regression was performed to assess all relationships between reported daily step count and four month change in WC. Results are reported as co-efficients representing the mean change in $\mathrm{WC}$ and were considered significant at a $\mathrm{p}$-value of less than 0.05 . Unadjusted regression was first completed followed by adjustment for age, sex and education (model 1) and adjustment for age, sex, education and change in self-reported physical activity during the workplace health program (model 2, Table 3) or baseline WC guidelines (model 2, Table 4). All analyses were clustered by workplace and performed using Stata version 10 (Stata Corporation, College Station, TX).

\subsection{Ethics}

The study, project number CF08/0271-2008000125, was approved by Monash University Human Research Ethics through the Standing Committee on Ethics in Research involving Humans and included the process of informed consent.

\section{RESULTS}

Descriptive statistics of the study population are presented in Table 1. Of the 498 participants included in the analysis, $326(65.4 \%)$ participants completed on average more than 10,000 steps per day throughout the four month health program (overall mean number of steps for the study population included herein 11,551). There was no significant difference in baseline age, sex, education, mean $\mathrm{WC}$, the proportion of participants meeting $\mathrm{WC}$ guidelines, or in the mean change in $\mathrm{WC}$ between those that completed less or more than 10,000 steps. Participants who completed an average of more than 10,000 steps were more likely to report an increase in physical activity during the health program.

\section{Change in Waist Circumference}

Overall, a significant decrease in WC of $-1.61 \mathrm{~cm}$ (95\% CI: $-2.13,-1.09)$ was observed between baseline and completion of the workplace program. No relationship between reported daily step count and change in WC was observed, regardless of whether reported daily step count was analysed as a continuous variable, a dichotomous variable or according to the step-defined physical activity hierarchy (Table 2).

Table 3 describes the relationship between reported daily step count and change in WC, stratified according to whether participants were meeting guidelines for WC at baseline. When examining this relationship using reported daily step count as a continuous variable, a relationship was observed between daily step count and change in WC in those not meeting WC guidelines at baseline, with a reduction of $-0.15 \mathrm{~cm}$ per 1000 steps (95\% CI: $-0.25,-0.05)$. No relationship was observed in those who met WC guidelines at baseline. This relationship was not appreciably affected by adjustment for perceived change in physical activity. In those not meeting WC guidelines at baseline, those achieving on average 10,000 steps or more per day improved their WC by $-1.24 \mathrm{~cm}(95 \% \mathrm{CI}:-2.25,-0.23)$ more than those not achieving 10,000 steps. This relationship reduced in magnitude and lost significance after adjustment for perceived change in physical activity.

Amongst participants who reported doing more physical activity during the workplace health program, a greater reported daily step count was associated with a greater reduction in WC (Table 4). When examining this relationship using reported daily step count as a continuous variable, each extra 1000 steps walked was associated with a $-0.22 \mathrm{~cm}(95 \% \mathrm{CI}:-0.36,-0.09)$ greater reduction in WC. When further dichotomised into step goal categories, there was a $-1.38 \mathrm{~cm}(95 \% \mathrm{CI}:-2.14$, -0.63 ) greater reduction in WC among participants who walked more than 10,000 steps when compared to those 
Table 1. Descriptive characteristics of study participants (mean or percentage and $95 \%$ confidence interval).

\begin{tabular}{lcc}
\hline & $<10,000$ steps & $\geq 10,000$ steps \\
\hline $\mathrm{n}$ & 142 & 294 \\
Age & $39.6(36.6,42.5)$ & $41.5(38.4,44.6)$ \\
Sex (males) & $40.8 \%(26.0,54.3)$ & $46.9 \%(32.7,59.3)$ \\
Tertiary education (degree or higher) & $83.0 \%(75.0,91.1)$ & $76.0 \%(71.0,81.2)$ \\
Baseline waist circumference (cm) & $88.7(87.2,90.1)$ & $87.6(85.4,89.9)$ \\
Meeting waist circumference guidelines at baseline & $44.8 \%(34.8,54.7)$ & $48.2 \%(41.2,55.1)$ \\
Change in waist circumference (cm) & $-1.6(-2.2,0.9)$ & $-1.6(-2.9,-0.4)$ \\
Meeting physical activity guidelines at baseline & $49.1 \%(40.8,54.2)$ \\
Reported an increase in physical activity during program $^{*}$ & $27.5 \%(14.6,34.5)$ & $56.5 \%(41.1,71.8)$ \\
Reported average daily step count $^{*}$ & $43.0 \%(27.9,58.0)$ & $13518(13150,13886)$ \\
\hline
\end{tabular}

*Demonstrated a significant difference between step categories with $\mathrm{p} \leq 0.05$.

Table 2. Change in waist circumference associated with reported daily step count (mean and $95 \%$ confidence interval).

\begin{tabular}{|c|c|c|c|c|}
\hline & n & $\begin{array}{l}\text { Change in waist } \\
\text { circumference }(\mathrm{cm})\end{array}$ & Univariate & Model $1 \wedge$ \\
\hline \multirow{3}{*}{ Continuous steps } & & & \multicolumn{2}{|c|}{ Waist circumference change (cm/1000 steps) } \\
\hline & 436 & - & $-0.04(-0.19,0.10)$ & $-0.03(-0.19,0.13)$ \\
\hline & & & \multicolumn{2}{|c|}{ Waist circumference change (cm) } \\
\hline \multicolumn{5}{|l|}{10,000 step categories } \\
\hline$<10,000$ steps & 172 & $-1.55(-2.22,-0.88)$ & reference & reference \\
\hline$\geq 10,000$ steps & 326 & $-1.64(-2.89,-0.39)$ & $-0.09(-1.48,1.29)$ & $-0.16(-1.47,1.16)$ \\
\hline \multicolumn{5}{|l|}{$\begin{array}{l}\text { Step-defined physical activity } \\
\text { hierarchal categories }\end{array}$} \\
\hline $\begin{array}{l}\text { Sedentary }(<5000 \mathrm{step} / \text { day }) \text { or below } \\
\text { active }(5000-7499 \mathrm{steps} / \text { day })\end{array}$ & 53 & $-2.25(-3.52,-0.97)$ & $-1.00(-2.64,0.63)$ & $-0.84(-2.49,0.82)$ \\
\hline $\begin{array}{l}\text { Somewhat active } \\
\text { (7500 - } 9999 \text { steps/day) }\end{array}$ & 119 & $-1.24(-2.04,-0.45)$ & reference & reference \\
\hline Active $(10,000-12,499$ steps/day) & 162 & $-1.52(-2.92,-0.12)$ & $-0.28(-1.69,1.14)$ & $-0.35(-1.74,1.05)$ \\
\hline Highly active (>12,500 steps/day) & 164 & $-1.77(-2.98,-0.56)$ & $-0.53(-1.72,0.69)$ & $-0.49(-1.75,0.77)$ \\
\hline
\end{tabular}

${ }^{*}$ Demonstrated a significant difference between step categories with $\mathrm{p} \leq 0.05 ;{ }^{\wedge}$ Adjusted for age, sex and education.

who did not achieve the 10,000 daily step goal. The magnitude of this relationship was increased after adjustment for baseline WC guidelines. In those participants reporting no change or a decrease in physical activity levels during the workplace health program no relationship was observed between reported daily step count and change in WC.

The results did not differ appreciably in sensitivity analyses when reported daily step count was calculated to include estimated step counts for missing entries (data not shown).

\section{DISCUSSION}

In this evaluation of a four month, pedometer-based, workplace health program, no overall relationship was observed between the reported number of daily steps and change in WC. However, in those not meeting WC guidelines at baseline a greater reported daily step count was associated with a greater decrease in WC. Similarly, amongst individuals who perceived an increase in physical activity during the program, a greater reported daily step count was associated with a greater reduction in WC. 
Table 3. Change in waist circumference associated with reported daily step count according to waist circumference guidelines at baseline (mean and $95 \%$ confidence interval).

\begin{tabular}{|c|c|c|c|c|}
\hline $\mathbf{n}$ & $\begin{array}{l}\text { Change in waist } \\
\text { circumference }(\mathrm{cm})\end{array}$ & Univariate & Model 1^ & Model $2^{\#}$ \\
\hline
\end{tabular}

Waist circumference change (cm/1000 steps)

\begin{tabular}{|c|c|c|c|c|c|}
\hline \multicolumn{6}{|l|}{ Continuous steps } \\
\hline Meeting WC guidelines at baseline & 234 & & $-0.05(-0.08,0.18)$ & $-0.05(-0.12,0.22)$ & $-0.06(-0.16,0.27)$ \\
\hline Not meeting WC guidelines at baseline & 264 & & $-0.13(-0.24,-0.02)^{*}$ & $-0.15(-0.25,-0.05)^{*}$ & $-0.15(-0.25,-0.04)^{*}$ \\
\hline & & & \multicolumn{3}{|c|}{ Waist circumference change $(\mathbf{c m})$} \\
\hline \multicolumn{6}{|l|}{ Meeting WC guidelines at baseline } \\
\hline$<10,000$ steps & 77 & $-0.79(-1.89,0.31)$ & reference & reference & reference \\
\hline$\geq 10,000$ steps & 157 & $-0.10(-1.60,1.80)$ & $0.89(-1.01,2.79)$ & $0.83(-0.99,2.65)$ & $1.24(-1.45,3.94)$ \\
\hline \multicolumn{6}{|l|}{ Not meeting WC guidelines at baseline } \\
\hline$<10,000$ steps & 95 & $-2.17(-3.05,-1.29)$ & reference & reference & reference \\
\hline$\geq 10,000$ steps & 169 & $-3.26(-4.31,-2.22)$ & $-1.10(-2.22,0.03)$ & $-1.24(-2.25,-0.23)^{*}$ & $-0.98(-2.48,0.51)$ \\
\hline
\end{tabular}

"Demonstrated a significant difference between step categories with $\mathrm{p} \leq 0.05$; ^Adjusted for age, sex and education; ${ }^{*}$ Adjusted for age, sex, education and change in self-reported physical activity during the health program.

Table 4. Change in waist circumference associated with a change in physical activity during the workplace health program (mean and $95 \%$ confidence interval).

\begin{tabular}{|c|c|c|c|c|c|}
\hline & $\mathbf{n}$ & $\begin{array}{l}\text { Change in waist } \\
\text { circumference }(\mathrm{cm})\end{array}$ & Univariate & Model 1 & Model $2^{\#}$ \\
\hline & & & \multicolumn{3}{|c|}{ Waist circumference change (cm/1000 steps) } \\
\hline \multicolumn{6}{|l|}{ Continuous steps } \\
\hline $\begin{array}{l}\text { Less/same physical activity prior to } \\
\text { health program }\end{array}$ & 209 & & $0.19(-0.08,0.47)$ & $0.20(-0.09,0.50)$ & $0.13(-0.13,0.39)$ \\
\hline $\begin{array}{l}\text { More physical activity than prior to } \\
\text { health program }\end{array}$ & 227 & & $-0.23(-0.37,-0.08)^{*}$ & $-0.22(-0.36,-0.09)^{*}$ & $-0.23(-0.39,-0.07)^{*}$ \\
\hline & & & \multicolumn{3}{|c|}{ Waist circumference change (cm) } \\
\hline \multicolumn{6}{|l|}{$\begin{array}{l}\text { Less/same physical activity than } \\
\text { prior to GCC }\end{array}$} \\
\hline$<10,000$ steps & 81 & $-2.25(-3.95,-0.55)$ & reference & reference & reference \\
\hline$\geq 10,000$ steps & 124 & $-0.44(-2.51,1.62)$ & $1.81(-1.41,5.03)$ & $1.64(-1.35,4.64)$ & $1.28(-1.33,3.90)$ \\
\hline \multicolumn{6}{|l|}{$\begin{array}{l}\text { More physical activity than prior } \\
\text { to GCC }\end{array}$} \\
\hline$<10,000$ steps & 61 & $-1.53(-2.84,-0.22)$ & reference & reference & reference \\
\hline$\geq 10,000$ steps & 165 & $-2.89(-3.73,-2.06)$ & $-1.36(-2.25,-0.47)^{*}$ & $-1.38(-2.14,-0.63)^{*}$ & $-1.60(-2.34,-0.87)^{*}$ \\
\hline
\end{tabular}

${ }^{*}$ Demonstrated a significant difference between step categories with $\mathrm{p} \leq 0.05 ;{ }^{\wedge}$ Adjusted for age, sex and education; ${ }^{\#}$ Adjusted for age, sex, education and baseline waist circumference guidelines.

No relationship was observed between reported daily step count and change in $\mathrm{WC}$ in those meeting WC guidelines at baseline, nor in those reporting no change or a decrease in physical activity during the program.

In order to convey the appropriate motivational messages to participants of these pedometer-based health programs, it is important to understand the extent to which the actual reported daily step count versus simply increasing overall physical activity levels is important for improving health outcomes. Chan et al. revealed a positive relationship between the magnitude of increase in the number of steps per day and change in WC [15]. This 
dependent reduction in WC on an increase in physical activity levels is supported by the findings of Slentz et al (2004) who demonstrated a strong dose-response relationship between increasing amounts of physical activity and improvements in measures of central obesity [16]. In our study when examining the target of 10,000 steps per day, we observed greater reductions in WC amongst participants who reported more than 10,000 steps per day, but this was dependent on a concomitant increase in self-reported overall physical activity levels. Our results therefore suggest that an increase in physical activity levels and the actual level of steps attained may be important.

The dependency on an increase in physical activity levels to realise improvements in WC measures is not surprising if one considers the energy balance required for weight control. Similarly, it is not surprising that we did not observe greater reductions in WC amongst participants who reported more than 10,000 steps per day but reported the same or less overall physical activity levels. This group of individuals are likely to be made up of already highly active participants for whom a further increase in physical activity levels may not be needed, and in whom a decrease in WC is likely to be limited. Interestingly, we observed a large reduction in WC amongst participants who did not increase overall physical activity levels and reported less than 10,000 steps per day. This indicates that external factors other than change in overall physical activity levels may have contributed to improvements in WC. It is possible that simply being involved in the workplace health program increased awareness of other health measures such as diet composition and total energy intake that contributed to the reduction in WC observed among this group of participants. Additionally, it is possible that this group of individuals did increase their physical activity levels but did not perceive the change.

Our study also demonstrated that when analysing the relationship between reported daily step count and health outcomes it is important to identify those people with a higher risk status at baseline to ensure that there is the capacity to improve the outcome. The observed association between change in WC and reported number of steps among participants who were not meeting $\mathrm{WC}$ guidelines at baseline is encouraging, as a central aim of pedometer based workplace health programs is to improve risk factors in those who are at high risk. Although the relationship between continuous reported step count and WC among those not meeting WC guidelines at baseline was significant in all adjusted models, this was not the case when dichotomising steps into more or less than 10,000 steps. In this analysis the magnitude of association between steps and WC change was reduced and lost significance in the final model that additionally adjusted for perceived change in physical activity levels. It is possible that change in physical activity levels is more important than baseline risk when considering the association between step count and WC change, however due to limitations in the measurement accuracy of our physical activity change variable, it is difficult to ascertain this in the current study. Although the health benefits of an intervention are likely to be realised most in a high risk population as we demonstrate in our analysis, this does not preclude the importance of such programs to maintain good health among participants who are already healthy. As gradual weight gain is typical amongst middle aged adults [17] it is essential that pedometer based workplace interventions are also designed to maintain weight in already healthy participants. A primary motivation for participating in such programs are for health reasons [1]. Therefore, in order to manage participant expectations and prevent de-motivation through a lack of health benefits, health and step goals need to be individually targeted.

In this study we did not have the power to perform a finer dose response analysis of daily step count once we had stratified according to baseline risk and perceived change in physical activity levels. Alternatively, it is possible that the actual number of steps per se is not important and the change in physical activity levels, manifested through an increase in the reported daily step count, is more important. Future studies, with fine measures of change in physical activity levels, will be important to resolve whether there is a threshold number of steps required to achieve health benefits independent of the level of increase in physical activity.

The major limitation to this study is the lack of baseline step data and therefore the inability to objectively determine the magnitude of change in daily step count, and overall physical activity levels. An additional limitation is that although the pedometers used in this workplace health program are internally validated, they are not externally validated. However, the effect of both these limitations is likely to be misclassification, and therefore our observed interaction between change in PA and daily step count is likely to be an attenuation of any true effect.

The key strength of this evaluation study is the large sample size, the collection of objectively measured WC and the strong response rate at the four month follow-up, enabling us to undertake this exploratory analysis requiring a number of stratifications.

Increased levels of central adiposity has been found to be independently associated with an increased risk of diabetes [18-20], cardiovascular disease [21,22] and all cause mortality [23]. Thus it is important to understand the possible mechanisms for maximising the reduction in WC associated with such workplace health programs. 
Our results highlight that a greater average daily step count is effective for reducing $\mathrm{WC}$ in participants who are not meeting WC guidelines at baseline. We also highlight that it is important for workplace pedometer programs to realise that a step goal of 10,000 steps may not be associated with health improvement if it does not represent an increase in overall physical activity levels for the individual. Thus, the aim of workplace pedometer-based health programs should not only be focused around achieving a single, common step goal, but should also consider targeted step goals to encourage participants to increase overall physical activity levels. However, to fully disentangle the interaction between step count and changes in physical activity levels associated with improvements in WC, it is essential that future evaluations of pedometer-based workplace programs obtain detailed baseline step count information so that a change in physical activity levels as a result of the program can be determined.

In summary, this evaluation of a four month pedometer-based workplace health program demonstrated that in a general, working population, step count was not associated with change in WC during the program. However, among those who also increased overall physical activity levels during the program, and among those with high risk $\mathrm{WC}$ at the start of the program, a greater daily step count was associated with a greater reduction in WC during the program. Pedometer programs need to consider setting individual participant health goals and step goals in order to manage participant expectations about improvements in health markers.

\section{ACKNOWLEDGEMENTS}

We thank all the participants and workplaces involved in the study. We would like to acknowledge the Australian Research Council (ARC) and the Foundation for Chronic Disease Prevention ${ }^{\mathrm{TM}}$ in the Workplace, which is associated with the Global Corporate Challenge ${ }^{\circledR}$, for partially funding this study. Dr Anna Peeters is funded by a VicHealth Public Health Fellowship.

\section{DECLARATION OF COMPETING INTERESTS}

This study was partly funded by the Foundation for Chronic Disease Prevention $^{\mathrm{TM}}$ in the Workplace, which is associated with the Global Corporate Challenge ${ }^{\circledR}$. Neither the study design nor analysis design were influenced by the Foundation for Chronic Disease Prevention ${ }^{\mathrm{TM}}$ in the Workplace.

\section{REFERENCES}

[1] World Health Organization (2008) Preventing noncommunicable diseases in the workplace through diet and physical activity: WHO/World Economic Forum report of a joint event. WHO, Geneva.

[2] Proper, K.I., Koning, M., van der Beek, A.J., Hildebrandt, V.H., Bosscher, R.J. and van Mechelen, W. (2003) The effectiveness of worksite physical activity programs on physical activity, physical fitness, and health. Clinical Journal of Sport Medicine, 13, 106-117. doi:10.1097/00042752-200303000-00008

[3] Tudor-Locke, C., Williams, J.E., Reis, J.P. and Pluto, D. (2002) Utility of pedometers for assessing physical activity: convergent validity. Sports Medicine, 32, 795-808. doi:10.2165/00007256-200232120-00004

[4] Dugdill, L. and Springett, J. (2001) Evaluating health promotion programmes in the workplace. WHO Regional Publications (European Series), 92, 285-308.

[5] Bravata, D.M., Smith-Spangler, C., Sundaram, V., Gienger, A.L., Lin, N., Lewis, R., Stave, C.D., Olkin, I., Sirard, J.R. (2007) Using pedometers to increase physical activity and improve health: a systematic review. The Journal of the American Medical Association, 298, 2296-2304. doi:10.1001/jama.298.19.2296

[6] Richardson, C.R., Newton, T.L., Abraham, J.J., Sen, A., Jimbo, M., Swartz and A.M. (2008) A meta-analysis of pedometer-based walking interventions and weight loss. The Annals of Family Medicine, 6, 69-77. doi:10.1370/afm.761

[7] Cobiac, L.J., Vos, T. and Barendregt, J.J. (2009) Cost-effectiveness of interventions to promote physical activity: A modelling study. PLoS Medicine, 6, e1000110. doi:10.1371/journal.pmed.1000110

[8] Tudor-Locke, C. (2010) Steps to better cardiovascular health: How many steps does it take to achieve good health and how confident are we in this number? Current Cardiovascular Risk Reports, 4, 271-276. doi:10.1007/s12170-010-0109-5

[9] Freak-Poli, R., Wolfe, R., Backholer, K., de Courten, M. and Peeters, A. (2011) Impact of a pedometer-based workplace health program on cardiovascular and diabetes risk profile. Preventive Medicine, 53, 162-171. doi:10.1016/j.ypmed.2011.06.005

[10] Hatano, Y. (1993) Use of the pedometer for promoting daily walking exercise. Journal of the International Committee on Health, Physical Education and Recreation, 29, 4-8.

[11] Tudor-Locke, C., Hatano, Y., Pangrazi, R.P. and Kang, M. (2008) Revisiting "how many steps are enough?" Medicine \& Science in Sports \& Exercise, 40, S537-543. doi:10.1249/MSS.0b013e31817c7133

[12] Freak-Poli, W.R., Backholer, K., de Courten, M., Peeters, A. (2011) Change in cardiovascular and diabetes risk associated with participation in a four month workplace pedometer-based intervention. Preventive Medicine, in Press.

[13] National Health and Medical Research Council (2003) Clinical practice guidelines for the management of overweight and obesity in adults. Department of Health and Ageing of Australian Government, Canberra, 45-47.

[14] Tudor-Locke, C. and Bassett, D.R. Jr. (2004) How many steps/day are enough? Preliminary pedometer indices for 
public health. Sports Medicine, 34, 1-8. doi:10.2165/00007256-200434010-00001

[15] Chan, C.B., Ryan, D.A. and Tudor-Locke, C. (2004) Health benefits of a pedometer-based physical activity intervenetion in sedentary workers. Preventive Medicine, 39, 12151222. doi:10.1016/j.ypmed.2004.04.053

[16] Slentz, C.A., Duscha, B.D., Johnson, J.L., Ketchum, K., Aiken, L.B., Samsa, G.P., Houmard, J.A., Bales, C.W. and Kraus, W.E. (2004) Effects of the amount of exercise on body weight, body composition, and measures of central obesity. STRRIDE-A randomized controlled study. Archives of Internal Medicine, 164, 31-39. doi:10.1001/archinte.164.1.31

[17] Ball, K., Crawford, D., Ireland, P. and Hodge, A. (2003) Patterns and demographic predictors of 5-year weight change in a multi-ethnic cohort of men and women in Australia. Public Health Nutrition, 6, 269-281. doi:10.1079/PHN2002431

[18] Lundgren, H., Bengtsson, C., Blohme, G., Lapidus, L. and Sjostrom, L. (1989) Adiposity and adipose tissue distribution in relation to incidence of diabetes in women: Results from a prospective population study in Gothenburg, Sweden. International Journal of Obesity, 13, 413-423.

[19] Friedl, K.E. (2009) Waist circumference threshold values for type 2 diabetes risk. Journal of Diabetes Science and Technology, 3, 761-769.

[20] Wang, Y., Rimm, E.B., Stampfer, M.J., Willett, W.C. and $\mathrm{Hu}$, F.B. (2005) Comparison of abdominal adiposity and overall obesity in predicting risk of type 2 diabetes among men. The American Journal of Clinical Nutrition, 81, 555-563.

[21] Larsson, B., Svardsudd, K., Welin, L., Wilhelmsen, L., Bjorntorp, P. and Tibblin, G. (1984) Abdominal adipose tissue distribution, obesity, and risk of cardiovascular disease and death: 13 year follow up of participants in the study of men born in 1913. British Medical Journal (Clinical Research Edition), 288, 1401-1404. doi:10.1136/bmj.288.6428.1401

[22] Cameron, A.J., Dunstan, D.W., Owen, N., Zimmet, P.Z., Barr, E.L., Tonkin, A.M., Magliano, D.J., Murray, S.G., Welborn, T.A. and Shaw, J.E. (2009) Health and mortality consequences of abdominal obesity: evidence from the AusDiab study. Medical Journal of Australia, 191, 202208.

[23] Seidell, J.C. (2010) Waist circumference and waist/hip ratio in relation to all-cause mortality, cancer and sleep apnea. European Journal of Clinical Nutrition, 64, 35-41. doi:10.1038/ejen.2009.71 